\title{
HOLOMORPHIC PATH INTEGRALS IN TANGENT SPACE FOR FLAT MANIFOLDS
}

\author{
GUILLERMO CAPOBIANCO AND WALTER REARTES
}

\begin{abstract}
In this paper we study the quantum evolution in a flat Riemannian manifold. The holomorphic functions are defined on the cotangent bundle of this manifold. We construct Hilbert spaces of holomorphic functions in which the scalar product is defined using the exponential map. The quantum evolution is proposed by means of an infinitesimal propagator and the holomorphic Feynman integral is developed via the exponential map. The integration corresponding to each step of the Feynman integral is performed in the tangent space. Moreover, in the case of $S^{1}$, the method proposed in this paper naturally takes into account paths that must be included in the development of the corresponding Feynman integral.
\end{abstract}

\section{Contents}

1. Introduction

2. Quantization of the phase space

2.1. Metric and complex structure

2.2. Flat Riemannian manifolds

2.3. The Hilbert space

2.4. The $S^{1}$ case

3. Path Integral

4. Discussion

Acknowledgements

References

\section{IntRoduction}

The quantization of Riemannian manifolds is a very important topic in quantum mechanics in non-trivial spaces $[12,13,15,14,24]$. From a geometrical point of view, the quantization in the phase space is natural, mainly because of its relation to the geometric quantization. The geometric quantization is strongly linked to the symplectic structure of the phase space [28].

Of particular interest is the case of cotangent bundles of Riemannian manifolds. The cotangent bundle carries a natural Riemannian structure which is the lifting of the structure at the base. It also has a compatible almost-complex structure $J$.

Date: May 20, 2019.

2010 Mathematics Subject Classification. 53Z05, 81S40.

Key words and phrases. Holomorphic quantization, path integrals, reproducing kernel Hilbert spaces. 
This almost-complex structure gives rise to a complex structure if and only if the manifold is flat [3]. This is the case we deal with in this paper.

We propose a quantization method for a flat Riemannian manifold using the complex structure of the cotangent bundle to define Hilbert spaces of holomorphic functions defined in the cotangent. To define the scalar product in this Hilbert space we make use of the exponential map, which allows us to perform an integration in the tangent space of the cotangent. The procedure is as follows. We assign to each point of the cotangent a Hilbert space by defining a scalar product by integration in the tangent space using a Gaussian measure. These spaces are naturally isomorphic. With this product, each fiber is a Segal-Bargmann space for locally defined holomorphic functions. The space of globally defined holomorphic functions is a subspace of the former one. The space we get is a reproducing kernel Hilbert space (RKHS), like that of Segal-Bargmann. The existence of a reproducing kernel allows us to study the temporal evolution of the quantum wave function by means of Feynman integrals. Moreover, we develop a method that takes into account all the paths that appear in the Feynman integral.

In [26], the author studies how to propose a Feynman integral in non-simply connected configuration spaces that consider all the paths. For example, in the case of $S^{1}$ [26], the Green function obtained by studying a free particle, whose Lagrangian is $L=\frac{1}{2} \dot{\theta}^{2}$, is given by the following expression

$$
G\left(\theta, T, \theta_{0}\right)=\frac{1}{\sqrt{2 \pi i T}} e^{i \frac{\left(\theta-\theta_{0}\right)^{2}}{2 T}} .
$$

However, it does not turn out to be the Green function for $S^{1}$, considering that the correct Green function must take into account all the paths, that is to say

$$
G\left(\theta, T, \theta_{0}\right)=\sum_{\mathbb{Z}} G_{n}\left(\theta, T, \theta_{0}\right),
$$

where

$$
G_{n}\left(\theta, T, \theta_{0}\right)=\frac{1}{\sqrt{2 \pi i T}} e^{i \frac{\left(\theta-\theta_{0}+2 \pi n\right)^{2}}{2 T}},
$$

Then, a Green function is finally obtained which coincides with that resulting from the infinitesimal propagator proposed in [25]. In the construction of this propagator, the integration is performed in the tangent space of $S^{1}$.

In this paper we do the integration in the tangent space of the cotangent and therefore, for the aforementioned cases, all the paths are automatically considered.

\section{Quantization of the Phase space}

2.1. Metric and complex structure. In the case of a Riemannian manifold $Q$, as a configuration space, there is a metric in the cotangent space that is the natural lifting of the metric in $Q$. Also an almost-complex structure is obtained, which is compatible with the symplectic structure of the cotangent bundle. This structure is integrable when the curvature of $Q$ is null [3, 13].

The metric in $Q$ can be naturally lifted to the cotangent space $P$ as follows. (See the details in the Appendix of [1]).

We denote by $\sigma$ the metric on $Q$, and by $\sigma^{\sharp}$ the isomorphism induced by $\sigma$ between $P=T^{*} Q$ and $T Q$. Then, we have a metric $G$ on $P$ given by

$$
G_{m}(V, W)=\sigma_{q}(T \pi V, T \pi W)+\sigma_{q}\left(\sigma_{q}^{\sharp} \frac{D p_{1}}{\mathrm{~d} t}(0), \sigma_{q}^{\sharp} \frac{D p_{2}}{\mathrm{~d} t}(0)\right),
$$


where $T \pi$ is the tangent application of the projection $\pi: P \rightarrow Q$, and $D$ is the covariant derivative.

A third structure appears naturally, the almost-complex structure $J$. The triple $(J, G, \omega)$ is known as a compatible triple. That is, given the fields $V$ and $W$, they verify $G(V, W)=\omega(V, J W)$.

Complexifying the tangent space $T_{m} P$, we have the space $T_{m} P \otimes \mathbb{C}$. The map $J_{m}$ can be extended naturally, and we have the decomposition $T_{m} P \otimes \mathbb{C}=T_{m}^{(1,0)} P \oplus$ $T_{m}^{(0,1)} P$ for all $m \in P$ which can be extended to the whole fiber bundle $T P$. Then, the complexified tangent bundle, $T^{\mathbb{C}} P$, splits as follows (see [13])

$$
T^{\mathbb{C}} P=T^{(1,0)} P \oplus T^{(0,1)} P,
$$

where $T^{(1,0)} P$ and $T^{(0,1)} P$ are the images of the projections $\Pi^{+}$and $\Pi^{-}$given by $\Pi^{ \pm}=\frac{1 \mp \mathrm{i} J}{2}$.

The projection $\Pi^{+}$is a natural isomorphism between $T_{m} P$ and $T_{m}^{(1,0)} P$ (holomorphic tangent space). That is, given a vector $V=\left(\dot{q}^{1}, \ldots, \dot{q}^{n}, \dot{p}_{1}, \ldots, \dot{p}_{n}\right) \in T_{m} P$, $\Pi^{+} V=\dot{z}^{i} \frac{\partial}{\partial z^{i}}$, where the induced complex coordinates are

$$
\dot{z}^{i}=\dot{q}^{i}+\mathrm{i} \sigma^{i m}\left(\dot{p}_{m}-p_{k} \Gamma_{m l}^{k} \dot{q}^{l}\right),
$$

and the corresponding holomorphic vector fields are

$$
\frac{\partial}{\partial z^{i}}=\frac{1}{2}\left(\frac{\partial}{\partial q^{i}}+p_{k} \Gamma_{i j}^{k} \frac{\partial}{\partial p^{j}}-\mathrm{i} \sigma_{i j} \frac{\partial}{\partial p^{j}}\right),
$$

where $\sigma_{i j}$ and $\Gamma_{i j}^{k}$ are the matrix coefficients of the metric and the Christoffel symbols respectively (the Einstein summation convention is used). Computing the Lie bracket of the above-mentioned fields, we obtain

$$
\left[\frac{\partial}{\partial z^{i}}, \frac{\partial}{\partial z^{j}}\right]=\mathrm{i} R_{k i j}^{m} p_{m} \sigma^{l k}\left(\frac{\partial}{\partial z^{l}}-\frac{\partial}{\partial \bar{z}^{l}}\right) .
$$

Then, by the Nirenberg-Newlander theorem [13], the distribution is integrable if and only if the curvature tensor of the metric $\sigma$ is identically null, see [3]. That is, the base manifold is flat. In this paper we assume that the configuration space is a flat manifold.

Recently, the research on flat Riemannian manifolds (known as euclidean space forms $[27,16])$ has shown many developments. For instance, its spectral properties are studied in detail in [22, 23]. In cosmology, the euclidean space forms are used to model the spatial part of flat universe models [2, 17, 18, 19, 20]. And more recently a quantization procedure for euclidean space forms based on path integrals is developed in [1].

2.2. Flat Riemannian manifolds. The following theorem by W. Killing and H. Hopf characterizes flat Riemmanian manifolds (it is a part of a more general theorem [27]).

Theorem 1. Let $M$ be a Riemannian manifold of dimension $n \geq 2$ and zero curvature. Then $M$ is complete and connected if and only if it is isometric to the quotient $\mathbb{R}^{n} / \Gamma$ with $\Gamma \subset E(n)$, where $\Gamma$ acts freely and properly discontinuously.

These manifolds are known as Euclidean space forms and $E(n)$ is the Euclidean group (semidirect product of the groups $O(n)$ and $\mathbb{R}^{n}$ ). 
In one dimension these manifolds are the real line $\mathbb{R}$ and the circle $S^{1}$ while in dimension 2 there are five manifolds, the plane $\mathbb{R}^{2}$, the cylinder, the infinite Möebius strip, the torus and the Klein bottle. In dimension 3 there are 18 types, 10 of which are compact, 6 orientable, and 4 non-orientable [16, 27]. In higher dimensions the number grows significantly.

Every homogeneous Riemannian manifold is diffeomorphic to some Lie group but in general a space form is not necessarily homogeneous. In particular, when the space form is homogeneous of dimension $n$, then it is isometric to the product $\mathbb{R}^{m} \times T^{n-m}$ of a Euclidean space with a flat Riemannian torus [27, p. 88].

This paper focuses on orientable compact flat manifolds. Compact flat Riemannian manifolds of dimension $n$ are quotients of polyhedra in $\mathbb{R}^{n}$ by identifying faces (see [27, p. 99]). Functions defined on the manifold are functions on $\mathbb{R}^{n}$, which are invariant under the action of the group.

An important invariant for a compact Euclidean space form is its volume. This can be defined in terms of a fundamental region for $\Gamma$ in $\mathbb{R}^{n}$ [21]. The volume of a space form $\mathbb{R}^{n} / \Gamma$ is defined to be the volume of any fundamental region, $c_{\gamma}$,

$$
c_{\gamma}:=\left\{x \in \mathbb{R}^{n} ;\|\gamma(0)-x\| \leq\left\|\gamma^{\prime}(0)-x\right\| \text { for every } \gamma^{\prime} \in \Gamma\right\},
$$

where $\gamma(0)$ is the action of $\gamma$ on $0 \in \mathbb{R}^{n}$. $c_{\gamma}$ is an $n$-dimensional convex polyhedron in $\mathbb{R}^{n}$ bounded by hyperplanes which are perpendicular bisectors of line segments $\left[\gamma(0), \gamma^{\prime}(0)\right]$. Its boundary $\partial c_{\gamma}$ carries a locally finite decomposition into convex polyhedra of dimension $n-1$. The space form $\mathbb{R}^{n} / \Gamma$ is then obtained from $c_{\gamma}$ by identifying points in $\partial c_{\gamma}$ which are equivalent modulo $\Gamma$.

The family $\left\{c_{\gamma}\right\}$ forms a crystalline structure (see [27, p. 100]), that can be generated by translation of a finite set of vectors defining the crystal lattice. This set forms a basis of $\mathbb{R}^{n}$. Dual basis vectors multiplied by $2 \pi$ are the basis of the reciprocal lattice, $\mathcal{L}$. Let $K$ be an element of the reciprocal lattice, then a function with the symmetry of this lattice has a Fourier expansion given by

$$
f(x)=\sum_{K \in \mathcal{L}} c_{K} \mathrm{e}^{\mathrm{i} K \cdot x} .
$$

This function is well defined on the manifold if it is also invariant under the action of $\Gamma$, i.e., $(\gamma f)(x)=f(\gamma x)=f(x)$ for all $\gamma \in \Gamma$.

The 3-dimensional orientable compact Euclidean space forms present a particular interest for cosmology, since they could model the spatial part of the flat-universe models [2]. Recently J. Levin et al. seek to develop a plausible cosmological model using orientable compact Euclidean space forms of dimension 3 in agreement with results of observations made on the cosmic microwave background radiation [17, 18, 19, 20].

2.3. The Hilbert space. The cotangent bundle $P$ with the metric $G$ is a complete Riemannian manifold. Then, by the theorem of Hopf-Rinow, it is geodesically complete, that is, for all $m \in P$ there exists a well defined exponential map on the entire tangent space $T_{m} P$.

Here, the tangent vectors are represented by its complex coordinates (5) using the projection $\Pi^{+}$. The square modulus $|z|^{2}$ is given by

$$
|z|^{2}=G_{m}(z, z)=\sigma_{i j}(\pi m) z^{i} \bar{z}^{j}
$$

where $\sigma_{i j}$ are the components of the matrix corresponding to the metric in the base manifold $Q$. 
The natural volume in the vectorial space $T_{m} P$ is given by the pull-back of the Riemannian metric by the exponential map (which coincides with the Liouville volume).

Proposition 1. The pullback of the Riemannian volume by the exponential map in $m$ is given by

$$
\exp _{m}^{*} \Omega(z)=\sigma(\pi m) d z \text {. }
$$

where $\sigma$ is the determinant of the metric, and $d z$ is the Lebesgue measure in $\mathbb{C}^{n}$.

We use the exponential map in order to define a product of holomorphic functions as follows.

Definition 1. Let $m \in P$, then for holomorphic functions $\phi$ and $\psi$ defined on $P$ we def ine the following normalized scalar product

$$
\langle\phi, \psi\rangle=\int_{T_{m} P} \overline{\phi\left(\exp _{m} z\right)} \psi\left(\exp _{m} z\right) e^{-|z|^{2}} d z .
$$

Even though the definition depends on the point $m$, the different products are isometric to each other. In the following theorem we demonstrate that all these elements constitute a Hilbert space of holomorphic functions.

Theorem 2. Let $Q$ be a flat Riemannian manifold, connected and geodesically complete. We assume the surjectivity of the exponential map. Then, the space of square integrable holomorphic functions on $P$ is a Hilbert space with the norm associated to the scalar product (9). We call this Hilbert space $\mathcal{B}_{P}$.

Proof. A holomorphic function $\phi: P \rightarrow \mathbb{C}$ induces (by pull-back) a holomorphic function on $T_{m} P$, that is $\tilde{\phi}=\phi \circ \exp _{m}$. The set of all holomorphic functions on $T_{m} P$ whit the scalar product $(9)$ is a Hilbert space $\mathcal{B}=\mathcal{H} L^{2}\left(\mathbb{C}^{n}, e^{-|z|^{2}}\right) \subset L^{2}\left(\mathbb{C}^{n}, e^{-|z|^{2}}\right)$ (see [9]). Let $\left\{\phi_{i}\right\}_{i=1}^{\infty}$ be a Cauchy sequence of holomorphic functions on $P$. It induces a new sequence $\left\{\tilde{\phi}_{i}\right\}_{i=1}^{\infty}$ which converges to a holomorphic function $\tilde{\phi}$ on $\mathcal{B}$. One of the key properties of these spaces of holomorphic functions is the continuity of the evaluation map, i. e., for all $z$ exists a constant $M_{z}$ such that for all $\tilde{\phi}$ it is verified that

$$
|\tilde{\phi}(z)|^{2} \leq M_{z}\|\tilde{\phi}\|_{L^{2}}^{2}
$$

Then,

$$
\left|\tilde{\phi}_{i}(z)-\tilde{\phi}(z)\right|^{2} \leq M_{z}\left\|\tilde{\phi}_{i}-\tilde{\phi}\right\|_{L^{2}}^{2} \rightarrow 0 \text {. }
$$

Then for all $n \in P$ we define $\phi(n)=\tilde{\phi}\left(\exp _{m}^{-1} n\right)$. The function $\phi$ is well defined. Indeed, if $z_{1}, z_{2} \in \exp _{m}^{-1} n$, we have $\tilde{\phi}_{i}\left(z_{1}\right)=\tilde{\phi}_{i}\left(z_{2}\right)$, then by $(11), \tilde{\phi}\left(z_{1}\right)=\tilde{\phi}\left(z_{2}\right)$.

We can consider $\mathcal{B}_{P}$ as a subspace of $\mathcal{B}$ by means of the exponential map. In $\mathcal{B}$ exists a function called reproducing kernel $K(z, w)$ (see [9]) with the following property

$$
\tilde{\phi}(z)=\int_{\mathbb{C}^{n}} K(z, w) \tilde{\phi}(w) e^{-|w|^{2}} d w
$$

for all $\tilde{\phi} \in \mathcal{B}$. Also $K(w, z)=\overline{K(z, w)}$ and satisfies the following composition rule

$$
K(z, u)=\int_{\mathbb{C}^{n}} K(z, w) K(w, u) e^{-|w|^{2}} d w
$$


for all $z, u \in \mathbb{C}^{n}$. Also for all $z \in \mathbb{C}^{n},|\tilde{\phi}(z)|^{2} \leq K(z, z)\|\tilde{\phi}\|^{2}(K(z, z)$ is optimal, that is, for each $z$, there exists a non-zero $\tilde{\phi}_{z} \in \mathcal{B}$ for which equality holds). Moreover, the reproducing kernel acts as a projector, i. e., if $\tilde{\phi} \in L^{2}\left(\mathbb{C}^{n}, e^{-|z|^{2}}\right)$, and calling $\mathcal{P} \tilde{\phi}$ to the orthogonal projection of $\tilde{\phi}$ onto $\mathcal{B}$, then

$$
\mathcal{P} \tilde{\phi}(z)=\int_{\mathbb{C}^{n}} K(z, w) \tilde{\phi}(w) e^{-|w|^{2}} d w .
$$

The reproducing kernel $K(z, w)$ is unique in the following sense. Given any $z \in \mathbb{C}^{n}$, if $F_{z}(\cdot) \in \mathcal{B}$ satisfies

$$
\tilde{\phi}(z)=\int_{\mathbb{C}^{n}} \overline{F_{z}(w)} \tilde{\phi}(w) e^{-|w|^{2}} d w
$$

for all $\tilde{\phi} \in \mathcal{B}$, then $\overline{F_{z}(w)}=K(z, w)$.

But in general the reproducing kernel $K(z, w)$ is not the pull-back of a function on $P$.

A reproducing kernel in the space $\mathcal{H}_{P}$, is a function

$$
K_{P}: P \times P \rightarrow \mathbb{C},
$$

which is holomorphic in the first coordinate and antiholomorphic in the second, and such that the following equation holds for all $\phi \in \mathcal{B}_{P}$

$$
\phi(n)=\int_{T_{m} P} K_{P}\left(n, \exp _{m} w\right) \phi\left(\exp _{m} w\right) e^{-|w|^{2}} d w .
$$

The following theorem allows us to obtain the reproducing kernel

Theorem 3. Let $\left\{e_{j}\right\}_{j=1}^{\infty}$ be an orthonormal basis for $\mathcal{B}_{P}$, then for $m, n \in P$

$$
\sum_{j=1}^{\infty}\left|e_{j}(m) \overline{e_{j}(n)}\right|<\infty
$$

and

$$
K_{P}(m, n)=\sum_{j=1}^{\infty} e_{j}(m) \overline{e_{j}(n)}
$$

Proof. Given that $\mathcal{B}_{P}$ is a Hilbert subspace of $\mathcal{B}$, we have the following direct sum

$$
\mathcal{B}=\mathcal{B}_{P} \oplus \mathcal{B}_{P}^{\perp}
$$

Let $\left\{f_{j}\right\}_{j=1}^{\infty}$ be an orthonormal basis of $\mathcal{B}$ compatible with this decomposition. Then, there exists a reproducing kernel $K(z, w)$ in this space (see [9]) given by

$$
K(z, w)=\sum_{j=1}^{\infty} f_{j}(z) \overline{f_{j}(w)} \text {. }
$$

Then we have

$$
K(z, w)=\tilde{K}_{P}(z, w)+\tilde{K}_{P}^{\perp}(z, w) .
$$

Considering that $\tilde{K}_{P}^{\perp}(z, w)$ acting on a function $\phi \in \mathcal{B}_{P}$ does not contribute to the integral, we have the following result

$$
\begin{aligned}
\phi(n) & =\int_{T_{m} P} K\left(\exp _{m}^{-1} n, w\right) \phi\left(\exp _{m} w\right) e^{-|w|^{2}} d w \\
& =\int_{T_{m} P} \tilde{K}_{P}\left(\exp _{m}^{-1} n, w\right) \phi\left(\exp _{m} w\right) e^{-|w|^{2}} d w
\end{aligned}
$$


and $K_{P}(n, q)=\tilde{K}_{P}\left(\exp _{m}^{-1} n, \exp _{m}^{-1} q\right)$ is the appropriate reproducing kernel.

2.4. The $S^{1}$ case. Consider the case of a particle whose configuration space is the unit circle. Then the phase space is a cylinder. The euclidean metric on the circle induces a euclidean metric on the cylinder. The system is interesting due to the non trivial topology, in particular it is a non-simply connected manifold. We choose the following coordinates

$$
-\pi<q<\pi \quad \text { y } \quad-\infty<p<\infty .
$$

And the following coordinates on the tangent space of the cylinder at the point $(0,0)$, called $x$ and $y$ respectively. That is, we can write a vector as follows

$$
V=x \frac{\partial}{\partial q}+y \frac{\partial}{\partial p} \text {. }
$$

The exponential map sends the point $(x, y)$ to the point $q=x \bmod 2 \pi$ and $p=y$ on the previous coordinate chart. In the tangent space of the cylinder we choose the following complex coordinates

$$
\begin{gathered}
z=x-i y \\
\bar{z}=x+i y .
\end{gathered}
$$

Now we consider the space of holomorphic functions on the cylinder, lifted to the tangent space by the exponential map. They are holomorphic in $z$ and periodic in the real part, $x$ coordinate. Then the scalar product in the space of holomorphic functions on the cylinder is

$$
\langle\phi, \psi\rangle=\frac{1}{2 \pi} \int_{\mathbb{C}} \overline{\phi(z)} \psi(z) e^{-z \bar{z}} d z .
$$

This space is similar to the Segal-Bargmann space, moreover, the functions are periodic in the real part. In view of theorem 2, it is a Hilbert subspace of $\mathcal{B}$. Actually, in the expression (27), the functions $\phi$ and $\psi$ in the integral are the functions $\phi$ and $\psi$ lifted to the tangent space by the exponential map respectively. For simplicity we call they the same way. With this scalar product the set of square integrable holomorphic functions on the cylinder is a Hilbert space. We call it $\mathcal{H}_{P}$.

A function in $\mathcal{H}_{P}$ has the following form

$$
\psi(z)=\sum_{n=-\infty}^{\infty} c_{n} e^{i n z}
$$

The functions

$$
\phi_{n}(z)=e^{i n z}=e^{i n(x-i y)}, \quad n \in \mathbb{Z}
$$

are periodic in the coordinate $x$ with period $2 \pi$. The set $\left\{\phi_{n}\right\}$ is a non-orthogonal basis of $\mathcal{H}_{P}$, in fact

$$
\left\langle\phi_{p}, \phi_{q}\right\rangle=e^{p q} .
$$

Considering that $\left\|\phi_{p}\right\|=e^{p^{2} / 2}$, then we have the normalized functions $\tilde{\phi}_{p}$

$$
\tilde{\phi}_{p}(z)=\frac{\phi_{p}(z)}{\left\|\phi_{p}\right\|}=e^{i p z-p^{2} / 2}
$$

With these functions, the scalar product is

$$
\left\langle\tilde{\phi}_{p}, \tilde{\phi}_{q}\right\rangle=e^{-(p-q)^{2} / 2} \text {. }
$$


2.4.1. Reproducing kernel. It can be directly verified that the reproducing kernel is

$$
K(z, \bar{w})=\frac{1}{2 \pi} \int_{-\pi}^{\pi} \frac{\rho_{t}^{z}(x) \rho_{t}^{\bar{w}}(x)}{\rho_{t}^{x_{0}}(x)} \mathrm{d} x
$$

where

$$
\rho_{t}^{z}(x)=\frac{1}{2 \pi} \sum_{k \in \mathbb{Z}} e^{i k(x-z)-k^{2} \frac{t}{2}}
$$

is the solution of the heat equation on $S^{1}$ with $t=1[4,5]$.

That is, given a function $\phi(z)$ as in (28), it verifies (12), i. e.

$$
\phi(z)=\sum_{k \in \mathbb{Z}} \phi_{k} e^{i k z}=\int_{\mathbb{C}} K(z, \bar{w}) \phi(w) e^{-|w|^{2}} d w
$$

Indeed,

$$
\begin{aligned}
\phi(z) & =\frac{1}{(2 \pi)^{4}} \int_{\mathbb{C}} \int_{-\pi}^{\pi} \frac{1}{\rho_{1}^{x_{0}}(x)} \sum_{k, l, m \in \mathbb{Z}} e^{-i k(x-z)-\frac{k^{2}}{2}} e^{i l(x-\bar{w})-\frac{l^{2}}{2}} \phi_{m} e^{i m w-w \bar{w}} d w \\
& =\sum_{k \in \mathbb{Z}} \phi_{k} e^{i k z}
\end{aligned}
$$

It is interesting to note that the reproducing kernel $K(z, \bar{w})$ coincides with that found in [1], although in that paper the reproducing kernel is found using a generalized Segal-Bargmann transform $[9,6,7,8,10,11]$ and the scalar product in that case is defined by an integration in the manifold, in which integrand the solution of the heat equation explicitly appears.

2.4.2. Ladder Operators. Now we can define the ladder operators $a^{+}$and $a$ (creation operator and annihilation operator respectively).

Given an orthonormal basis $\left\{\beta_{i}\right\}$ of $\mathcal{H}_{P}$, we have the following expression for the reproducing kernel

$$
K(z, \bar{w})=\sum_{n=0}^{\infty} \beta_{n}(z) \overline{\beta_{n}(w)}=\overline{\zeta_{\bar{z}}(w)} .
$$

And the operators are the following

$$
a^{+} \psi(z)=\left\langle\zeta_{\bar{z}}, w \psi\right\rangle_{L^{2}}=\frac{1}{2 \pi} \sum_{n=0}^{\infty} \beta_{n}(z) \int_{\mathbb{C}} \overline{\beta_{n}(w)} w \psi(w) e^{-w \bar{w}} d w
$$

and

$$
a \chi(z)=\left\langle\zeta_{\bar{z}}, \frac{d \chi}{d w}\right\rangle_{L^{2}}=\frac{1}{2 \pi} \sum_{n=0}^{\infty} \beta_{n}(z) \int_{\mathbb{C}} \overline{\beta_{n}(w)} \frac{d \chi}{d w}(w) e^{-w \bar{w}} d w=\frac{d \chi}{d z}(z) .
$$

The functions $\psi$ and $\chi$ are given by the following series

$$
\psi(z)=\sum_{p=0}^{\infty} u_{p} \beta_{p}(z), \quad \chi(z)=\sum_{q=0}^{\infty} v_{q} \beta_{q}(z) .
$$

Then

$$
\left\langle a^{+} \psi, \chi\right\rangle=\frac{1}{(2 \pi)^{2}} \sum_{n=0}^{\infty} \int_{\mathbb{C}} \overline{w \psi(w)} \beta_{n}(w) e^{-w \bar{w}} d w \int_{\mathbb{C}} \overline{\beta_{n}(z)} \chi(z) e^{-z \bar{z}} d z
$$




$$
=\frac{1}{(2 \pi)^{2}} \sum_{n=0}^{\infty} \int_{\mathbb{C}} \overline{\psi(w)} \frac{d \beta_{n}}{d w}(w) e^{-w \bar{w}} d w \int_{\mathbb{C}} \overline{\beta_{n}(z)} \chi(z) e^{-z \bar{z}} d z,
$$

where the last expression was obtained integrating by parts. Then, using the series expansion of the functions $\psi$ and $\chi$, we obtain

$$
\begin{aligned}
\left\langle a^{+} \psi, \chi\right\rangle & =\frac{1}{(2 \pi)^{2}} \sum_{n, p, q=0}^{\infty} \bar{u}_{p} v_{q} \int_{\mathbb{C}} \frac{d \beta_{n}}{d w}(w) \overline{\beta_{p}(w)} e^{-w \bar{w}} d w \int_{\mathbb{C}} \overline{\beta_{n}(z)} \beta_{q}(z) e^{-z \bar{z}} d z \\
& =\frac{1}{2 \pi} \sum_{p, q=0}^{\infty} \bar{u}_{p} v_{q} \int_{\mathbb{C}} \frac{d \beta_{q}}{d w}(w) \overline{\beta_{p}(w)} e^{-w \bar{w}} d w
\end{aligned}
$$

where we use the orthonormality of $\beta_{i}$ 's.

Also

$$
\langle\psi, a \chi\rangle=\frac{1}{(2 \pi)^{2}} \sum_{n=0}^{\infty} \int_{\mathbb{C}} \overline{\beta_{n}(w)} \frac{d \chi}{d w}(w) e^{-w \bar{w}} d w \int_{\mathbb{C}} \overline{\psi(z)} \beta_{n}(z) e^{-z \bar{z}} d z .
$$

where we use again the series expansion of $\psi$ and $\chi$,

$$
\begin{aligned}
\langle\psi, a \chi\rangle & =\frac{1}{(2 \pi)^{2}} \sum_{n, p, q=0}^{\infty} \bar{u}_{p} v_{q} \int_{\mathbb{C}} \frac{d \beta_{q}}{d w}(w) \overline{\beta_{n}(w)} e^{-w \bar{w}} d w \int_{\mathbb{C}} \overline{\beta_{p}(z)} \beta_{n}(z) e^{-z \bar{z}} d z \\
& =\frac{1}{2 \pi} \sum_{p, q=0}^{\infty} \bar{u}_{p} v_{q} \int_{\mathbb{C}} \frac{d \beta_{q}}{d w}(w) \overline{\beta_{p}(w)} e^{-w \bar{w}} d w .
\end{aligned}
$$

Then we have the following result

$$
\left\langle a^{+} \psi, \chi\right\rangle=\langle\psi, a \chi\rangle,
$$

i. e., $a^{+}$and $a$ are adjoint operators.

\section{Path Integral}

Let $Q$ be a Riemannian manifold, connected and geodesically complete. Let $P$ be the cotangent bundle of $Q$. In section (2.1) we shown that there is a natural lifting of the metric $\sigma$ in $Q$ to a metric $G$ in $P$ (see (4)). Also, every simplectic manifold is almost-Kähler, then $P$ admits a compatible almost-complex structure $J$. We choose $J$ such that the metric $G=\omega(\cdot, J \cdot)$ coincides with the lifting of the metric in $Q$. If $J$ is integrable, $P$ is a Kähler manifold.

Now we study the temporal evolution of the quantum wave function. Let $K: P \times$ $P \rightarrow \mathbb{C}$ be the reproducing kernel, then

$$
\phi(m)=\int_{T_{m} P} K\left(m, \exp _{m} z\right) \phi\left(\exp _{m} z\right) e^{-|z|^{2}} d z .
$$

Let $H$ be the hamiltonian operator in $\mathcal{H} L^{2}(P)$, represented by the integral kernel $K_{H}$, i. e.

$$
H \phi(m)=\int_{T_{m} P} K_{H}\left(m, \exp _{m} z\right) \phi\left(\exp _{m} z\right) e^{-|z|^{2}} d z .
$$

According to the solution of the Schrödinger equation, the evolution operator associated to time-independent hamiltonians is

$$
U_{t}=e^{-i H t} \text {. }
$$


For small time intervals we have the following approximation

$$
U_{\Delta} \cong 1-i H \Delta \text {. }
$$

Then, we define the infinitesimal evolution operator as follows, [1]

$$
\begin{gathered}
u_{\Delta} \phi(m)=\int_{T_{m} P} K\left(m, \exp _{m} z\right) \phi\left(\exp _{m} z\right) e^{-|z|^{2}} e^{-i \Delta K_{H} / K} d z . \\
u_{\Delta}\left(u_{\Delta} \phi\right)(m)=\int_{T_{m} P} K\left(m, \exp _{m} z\right) \int_{T_{\left(\exp _{m} z\right)} P} K\left(\exp _{m} z, \exp _{\left(\exp _{m} z\right)} z_{2}\right) \\
\phi\left(\exp _{\left(\exp _{m} z\right)} z_{2}\right) e^{-i \Delta K_{H}\left(\exp _{m} z, \exp _{\left(\exp _{m} z\right)} z_{2}\right) / K\left(\exp _{m} z, \exp _{\left(\exp _{m} z\right)} z_{2}\right)} \\
e^{-i \Delta K_{H}\left(m, \exp _{m} z\right) / K\left(m, \exp _{m} z\right)} e^{-\left|z_{2}\right|^{2}} d z_{2} e^{-|z|^{2}} d z .
\end{gathered}
$$

Now, we use the following notation

$$
\begin{gathered}
\exp _{m} z=m_{2} \\
\exp _{\left(\exp _{m} z\right)} z_{2}=m_{3} \\
\exp _{\left(\exp _{\left(\exp _{m} z\right)} z_{2}\right)} z_{3}=m_{4}
\end{gathered}
$$

In that way we can define in general $m_{j}$. It is important to note that $m_{j}=$ $m_{j}\left(z_{j}, z_{j-1}, \ldots, z_{2}, z\right)$. This allows us to write (49) as follows

$$
\begin{aligned}
& u_{\Delta}\left(u_{\Delta} \phi\right)(m)= \\
& \int_{T_{m} P} K\left(m, m_{2}\right) \int_{T_{m_{2}} P} K\left(m_{2}, m_{3}\right) \phi\left(m_{3}\right) e^{-i\left(\frac{\Delta K_{H}\left(m_{2}, m_{3}\right)}{K\left(m_{2}, m_{3}\right)}+\frac{\Delta K_{H}\left(m_{,} m_{2}\right)}{K\left(m, m_{2}\right)}\right)} e^{-\left|z_{2}\right|^{2}} d z_{2} e^{-|z|^{2}} d z .
\end{aligned}
$$

Then, after $n$ compositions, we have

$$
u_{\Delta}^{n} \phi(m)=\int_{T_{m} P} \ldots \int_{T_{m_{n}} P} \phi\left(m_{n+1}\right) e^{-i \sum_{i=1}^{n} \frac{\Delta K_{H}\left(m_{i}, m_{i+1}\right)}{K\left(m_{i}, m_{i+1}\right)}} \prod_{i=1}^{n} K\left(m_{i}, m_{i+1}\right) e^{-\left|z_{i}\right|^{2}} d z_{i} .
$$

Where in the last equation we identify $z$ as $z_{1}$ and $m$ as $m_{1}$ respectively. The composition of these infinitesimal evolution operators allows us to obtain the evolution operator for finite time $t$ as

$$
U_{t} \phi(m)=\lim _{n \rightarrow \infty}\left(u_{t / n}\right)^{n} \phi(m) .
$$

It is important to mention that the tangent space corresponding to a flat manifold is also a flat manifold, so in our evolution proposal we only deal with euclidean integrals in each step of the iteration.

Specifically, the six 3-dimensional orientable compact Euclidean space forms are the following quotient spaces $\mathbb{R}^{3} / \Gamma_{i}, i=1, \ldots, 6$ (see [27, p. 117] and [16, p. 302]). For example, for the torus $T^{3}$ (which is constructed by identifying the opposite faces of a parallelepiped by translations), $\Gamma_{1}$ is generated by three translations $t_{1}$, $t_{2}, t_{3}$, in the direction of three linear independent vectors. And $\Gamma_{3}$ is generated by $\Gamma_{1}$ and a screw motion $\alpha^{3}=t_{3}$. 
For instance, when the configuration space is the manifold $\mathbb{R}^{3} / \Gamma_{3}, P=T^{*}\left(\mathbb{R}^{3} / \Gamma_{3}\right)$ and

$$
\begin{aligned}
& u_{\Delta}^{n} \phi(m)= \\
& \int_{T_{m} T^{*}\left(\mathbb{R}^{3} / \Gamma_{3}\right)}^{\ldots} \int_{T_{m_{n}} T^{*}\left(\mathbb{R}^{3} / \Gamma_{3}\right)} \phi\left(m_{n+1}\right) e^{-i \sum_{i=1}^{n} \frac{\Delta K_{H}\left(m_{i}, m_{i+1}\right)}{K\left(m_{i}, m_{i+1}\right)}} \prod_{i=1}^{n} K\left(m_{i}, m_{i+1}\right) e^{-\left|z_{i}\right|^{2}} d z_{i},
\end{aligned}
$$

where $z_{i} \in \mathbb{C}^{3}$ and $\phi(m)$ is a function in $\mathbb{C}^{3}$ that preserves the symmetry of the euclidean space form and is invariant under the action of $\Gamma_{3}$.

\section{Discussion}

Recently, the research on flat Riemannian manifolds has shown many developments $[22,23,2,17,18,19,20]$. For instance, a quantization scheme for euclidean space forms based on path integrals is developed in [1].

In the present paper we make a different and original proposal to the quantization of flat manifolds. It is interesting to note that it shares some results (such as the reproducing kernel) with the scheme studied in [1].

Here we study the holomorphic quantization of a quantum state whose configuration space is a flat Riemannian manifold. The Hilbert spaces are obtained using the exponential map. To define the scalar product in this Hilbert space we make use of the exponential map, which allows us to perform an integration in the tangent space of the cotangent. Also the Feynman integral is developed via the exponential map. The existence of a reproducing kernel allows us to study the temporal evolution of the quantum wave function. As an advantage, the calculations are simpler. In [26], for example, the author studies the Feynman integral in non-simply connected configuration spaces and the obtained Green function must be corrected in order to include all the paths. In the case of $S^{1}$, the method proposed in our paper naturally takes into account all the paths.

Finally, in the last section we apply our quantization method to the case when the configuration space is the manifold $\mathbb{R}^{3} / \Gamma_{3}$, and we show the evolution operator in (53) as a composition of infinitesimal evolution operators.

Acknowledgements. This work was supported by the Universidad Nacional del Sur (Grants PGI 24/L096 and PGI 24/ZL10).

\section{REFERENCES}

1. Capobianco G., Reartes W.A., Path integrals on euclidean space forms, Symmetry, Integrability and Geometry: Methods and Applications (SIGMA) 11 (2015), 071, 12 pages.

2. Ellis G.F.R., Topology and cosmology, Gen. Relativity Gravitation 2 (1971), 7-21.

3. Gorbunov I.V., Lyakhovich S.L., Sharapov A.A., Wick quantization of cotangent bundles over Riemannian manifolds, J. Geom. Phys. 53 (2005), 98-121, hep-th/0401022.

4. Grigor'yan A., Heat kernels on weighted manifolds and applications, in The Ubiquitous Heat Kernel, Contemp. Math., Vol. 398, Editors J. Jorgenson, L. Walling, Amer. Math. Soc., Providence, RI, 2006, 93-191.

5. Grigor'yan A., Heat kernel and analysis on manifolds, AMS Studies in Advanced Mathematics, Vol. 47, Amer. Math. Soc., Providence, RI, 2009.

6. Hall B.C., The Segal-Bargmann "coherent state" transform for compact Lie groups, J. Funct. Anal. 122 (1994), 103-151. 
7. Hall B.C., The inverse Segal-Bargmann transform for compact Lie groups, J. Funct. Anal. 143 (1997), 98-116.

8. Hall B.C., Harmonic analysis with respect to heat kernel measure, Bull. Amer. Math. Soc. 38 (2001), 43-78, quant-ph/0006037.

9. Hall B.C., Holomorphic methods in analysis and mathematical physics, in First Summer School in Analysis and Mathematical Physics (Cuernavaca Morelos, 1998), Contemp. Math., Vol. 260, Editors S. Pérez-Esteva, C. Vilegas-Blas, Amer. Math. Soc., Providence, RI, 2000, 1-59, quant-ph/9912054.

10. Hall B.C., Kirwin W.D., Adapted complex structures and the geodesic flow, Math. Ann. 350 (2011), 455-474, arXiv:0811.3083.

11. Hall B.C., Mitchell J.J., The Segal-Bargmann transform for noncompact symmetric spaces of the complex type, J. Funct. Anal. 227 (2005), 338-371, quant-ph/0409118.

12. Kleinert H., Path integrals in quantum mechanics, statistics, polymer physics, and financial markets, 3rd ed., World Sci. Publ. Co., Inc., River Edge, NJ, 2004.

13. Kobayashi S., Nomizu K., Foundations of differential geometry. Vol. II, Interscience Tracts in Pure and Applied Mathematics, Vol. 15, John Wiley \& Sons, Inc., New York - London Sydney, 1969.

14. Kowalski K., Rembieliński J., Papaloucas L.C., Coherent states for a quantum particle on a circle, J. Phys. A: Math. Gen. 29 (1996), 4149-4167, quant-ph/9801029.

15. Kowalski K., Rembieliński J., Quantum mechanics on a sphere and coherent states, J. Phys. A: Math. Gen. 33 (2000), 6035-6048.

16. Kühnel W., Differential geometry. Curves - surfaces - manifolds, Student Mathematical Library, Vol. 16, Amer. Math. Soc., Providence, RI, 2002.

17. Levin J., Topology and the cosmic microwave background, Phys. Rep. 365 (2002), 251-333, gr-qc/0108043.

18. Levin J., Scannapieco E., de Gasperis G., Silk J., Barrow J.D., How the universe got its spots, Phys. Rev. D 58 (1998), 123006, 14 pages, astro-ph/9807206.

19. Levin J., Scannapieco E., Silk J., Is the universe infinite or is it just really big?, Phys. Rev. D 58 (1998), 103516, 5 pages, astro-ph/9802021.

20. Levin J., Scannapieco E., Silk J., The topology of the universe: the biggest manifold of them all, Classical Quantum Gravity 15 (1998), 2689-2697, gr-qc/9803026.

21. McMullen P., Schulte E., Abstract regular polytopes, Encyclopedia of Mathematics and its Applications, Vol. 92, Cambridge University Press, Cambridge, 2002.

22. Miatello R., Podestá R., Spectral properties of four-dimensional compact flat manifolds, Ann. Global Anal. Geom. 29 (2006), 17-50.

23. Miatello R., Rossetti J., Spectral properties of flat manifolds, Contemp. Math. 491 (2009), 83-113.

24. Mostafazadeh A., Scalar curvature factor in the Schrödinger equation and scattering on a curved surface, Phys. Rev. A 54 (1996), 1165-1170, hep-th/9602095.

25. Reartes W., Path integral quantization of the sphere, In New Advances in Celestial and Hamiltonian Systems, Kluwer Academic, New York (2004) 225-237.

26. Schulman L.S., Techniques and applications of path integration, Dover Publications, New York , 2005.

27. Wolf J.A., Spaces of constant curvature, 6th ed., AMS Chelsea Publishing, Providence, RI, 2011.

28. Woodhouse N.M.J., Geometric quantization, Clarenton Press, Oxford, 1991.

Departamento de Matemática, inmabB Conicet, Universidad Nacional del Sur, Av. Alem 1253, 8000 Bahía Blanca, Buenos Aires, Argentina

E-mail address: guillermo.capobianco@gmail.com,walter.reartes@gmail.com 